\title{
Revisión de estudios sobre metáforas conceptuales en ciencia y educación científica
}

\author{
A Review of Studies About Conceptual Metaphors in \\ Science and Science Education
}

\section{Iván Salinas Barrios}

Universidad de Chile

\section{Resumen}

\begin{abstract}
Esta revisión de literatura sintetiza los aportes de la investigación al estudio de metáforas conceptuales en educación científica. A propósito de la relevancia del fenómeno lingüístico de las metáforas conceptuales en el desarrollo de conceptos científicos, se hace necesario entender cómo ha influido la metáfora conceptual en el campo de estudios sobre aprendizaje y enseñanza de las ciencias. A partir de la teoría de metáfora conceptual, con la relevancia del experiencialismo y la tesis de la cognición incorporada, este trabajo presenta una revisión hermenéutica de 31 fuentes académicas publicadas principalmente en la última década sobre metáforas conceptuales en ciencias y educación científica. Se discuten e identifican algunos espacios para avanzar una agenda de investigación con potencialidad práctica y didáctica que involucre una mayor consideración sobre las relaciones entre el cuerpo humano, el ambiente, y el lenguaje en el aprendizaje escolar de las ciencias.
\end{abstract}

Palabras clave: metáforas conceptuales, aprendizaje de las ciencias, comprensión de conceptos.

\footnotetext{
Correspondencia a:

Iván Salinas Barrios

Departamento de Estudios Pedagógicos, Facultad de Filosofía y Humanidades,

Universidad de Chile.

Capitán Ignacio Carrera Pinto \#1025 Ñuñoa, Santiago, Chile 7800284.

iedusal@uchile.cl

Se agradece el apoyo financiero de ANID (Ex-CONICYT), programa FONDECYT 11170880 .

(C) 2021 PEL, http://www.pensamientoeducativo.org - http://www.pel.cl
} 


\title{
Abstract
}

\begin{abstract}
This literature review summarizes research contributions to the study of conceptual metaphors in science education. Given the importance of conceptual metaphors as a phenomenon in the development of scientific concepts, it is necessary to understand how they have influenced the field of studies on science learning and teaching. Based on the theory of conceptual metaphor and the relevance of experientialism and the embodied cognition thesis, this paper presents a hermeneutic review of 31 academic sources, mainly published in the last decade, about conceptual metaphors in science and science education. We identify and discuss spaces for advancing a research agenda with practical and teaching potential, with more consideration of the relationships between the human body, the environment, and language in school science learning.
\end{abstract}

Keywords: conceptual metaphors, science learning, conceptual understanding.

El psicolingüista Steven Pinker (2010), sugirió dos hipótesis para explicar por qué los humanos nos involucramos en tareas tan abstractas como la ciencia. La primera es que hemos desarrollado, a través de la evolución, un modo de sobrevivencia cuya característica principal es la manipulación del ambiente mediante el razonamiento causal y la cooperación social. Pinker denominó "nicho cognitivo" a esta idea de la interdependencia social y uso intensivo del conocimiento. La segunda es que las capacidades psicológicas que desarrollamos prósperamente en ese nicho cognitivo se pueden cooptar hacia dominios abstractos mediante procesos de metaforización, siendo el lenguaje su manifestación más vívida. La abstracción metafórica representa una clave con la que explicar las capacidades de razonamiento humano abstracto, incluyendo el razonamiento científico.

Si la abstracción metafórica es tan relevante para explicar el razonamiento científico, ¿cómo se ha involucrado esta perspectiva en la comunidad de investigación en educación científica? Este trabajo plantea una revisión de la literatura que busca responder esta pregunta y establecer la conexión entre la abstracción metafórica y el desarrollo de la educación científica.

La abstracción metafórica, o bien metáfora conceptual, es un fenómeno lingüístico bien notado en la literatura de las ciencias cognitivas -específicamente, la lingüística cognitiva-, siendo George Lakoff y Mark Johnson (Lakoff, 1993, 2008; Lakoff \& Johnson, 1980, 1999) sus pioneros exponentes en la década de 1980. La lingüística cognitiva es un campo de estudios que se enfoca en investigar la cognición mediante el examen del lenguaje (Croft \& Cruse, 2004; Evans \& Green, 2006; Geeraerts \& Cuyckens, 2007; Ibarretxe-Antuńano y Valenzuela, 2012; Lee, 2001; Ungerer \& Schmid, 1996). La lingüística cognitiva se basa en algunos supuestos básicos. El primero es que el lenguaje refleja propiedades fundamentales de la mente humana; el segundo, que la gramática en sí misma representa una conceptualización, y el tercero, que el conocimiento del y sobre el lenguaje emerge cuando se indaga el lenguaje en uso. De acuerdo con estos supuestos, el lenguaje en uso refleja la cognición, o bien el conocer algo sobre el lenguaje equivale a conocer algo respecto de procesos de significado, forma y pensamiento.

La metáfora conceptual es un fenómeno del lenguaje que se estudia con las herramientas de la lingüística cognitiva. Según Lakoff y Johnson (1980), el pensamiento metafórico es ubicuo e inevitable, y es fundamentalmente inconsciente. Desde esta perspectiva, un análisis de evidencia lingüística, del lenguaje en uso, podría proveer pistas respecto de las conceptualizaciones que tenemos - como humanos- sobre el mundo y sobre la ciencia. La metáfora conceptual puede ser parte de estas evidencias. Lakoff y Johnson (1980) enfatizaron que las metáforas conceptuales son diferentes a la noción convencional que las sitúa como ornamentos retóricos, estéticos o 
estilísticos del lenguaje. Esta visión ha permeado a otros campos de investigación aplicada (véase Geary, 2011), por lo que esta perspectiva ha motivado a investigadores en educación científica a explorar cuáles son estas metáforas conceptuales y cómo se vinculan con la comprensión y el aprendizaje de las ciencias. Este trabajo busca conectar las obras hasta ahora existentes en esta intersección de metáforas conceptuales, ciencias y educación científica, con el fin de entender las potencialidades de investigación y aplicación didáctica.

Para abordar la interrogante sobre el vínculo entre estudios de metáfora conceptual y educación científica, a continuación, y a modo de marco teórico, me refiero a las metáforas conceptuales y su relación con la corporalidad. Luego, presento una revisión de la literatura en relación con las metáforas conceptuales en ciencia y educación científica, para concluir con una discusión que aborda una agenda de investigación y las potencialidades didácticas.

\section{Marco teórico}

\section{Metáforas conceptuales, corporalidad y lenguaje}

Lakoff y Johnson (1980) describieron las metáforas como conceptuales, esenciales para el pensamiento abstracto. Una metáfora conceptual es un mapeo o apareamiento que va desde un dominio conceptual abstracto (dominio meta) a uno conceptual físico-material o una experiencia corporal (dominio fuente). El dominio meta es el concepto que se busca describir con la metáfora, mientras que el dominio fuente es aquel que sirve como origen para describir el dominio meta. En un dominio fuente, lo que prima son experiencias específicas, perceptuales y corporales y, a veces, culturales.

Dos conceptos de la lingüística cognitiva se relacionan con la idea de experiencias corporales: el experiencialismo y la tesis de la cognición incorporada. El experiencialismo afirma que el conocimiento humano sobre conceptos abstractos emerge de la experiencia humana con otros dominios de conocimiento, específicamente las experiencias corporales con el ambiente (Lakoff, 1987). La corporalidad se refiere a aquellas experiencias que derivan de las interacciones entre el cuerpo humano y el mundo físico y cultural. Estas interacciones son de carácter prelingüístico, o bien, ocurren antes de que el cuerpo humano pueda comunicarse mediante un lenguaje, y están íntimamente ligadas al desarrollo de la cognición (Lakoff \& Johnson, 1999). Experiencias sensoriales como la visión, el balance y la gravedad son ejemplos de corporalidad; también lo son experiencias emocionales como la rabia y la felicidad. La tesis de la cognición incorporada señala que los sistemas conceptuales de los humanos dependen de las formas en las que el cuerpo humano interactúa con el ambiente (Evans \& Green, 2006). Una consecuencia de asumir esta tesis es que la naturaleza del cuerpo humano y sus sistemas de percepción limitan, en la práctica, su capacidad de interactuar con el ambiente físico, y con ello el acceso a conceptualizaciones que provienen de estas interacciones (Lakoff \& Johnson, 1999). Así, los humanos solo tenemos acceso a pensar consecuentemente lo que el cuerpo humano permite percibir y concebir. Aun siendo de carácter prelingüístico, el lenguaje es capaz de capturar la naturaleza de las experiencias corporales y su influencia en la capacidad de conceptualización.

Considerando lo anterior, las relaciones del cuerpo con el ambiente serían responsables de establecer vínculos para la creación de dominios conceptuales fuente en las metáforas conceptuales. Una característica de las metáforas conceptuales es su unidireccionalidad en el mapeo entre los dominios que son mapeados. Esto implica que un dominio meta puede comprenderse como un dominio fuente, pero un dominio fuente no puede comprenderse como un dominio meta. Las siguientes expresiones, tomadas y adaptadas del corpus del espańol (Davies, 2017), ejemplifican esta unidireccionalidad:

- Sacaron a pasear al perro con una cadena de acero.

- Esa manipulación a la cifra global, provocó una cadena de errores.

- La doble cadena de ADN se separa. 
El primer ejemplo es una expresión lingüística sobre la percepción directa de una entidad, la cadena de acero, como vínculo entre una persona y una actividad específica, culturalmente situada, y corporalmente perceptible (representada en la figura 1(a)). La segunda expresión implica niveles de abstracción donde una cadena no es perceptible de la misma forma, sino que requiere de una operación de mapeo desde el dominio conceptual de una cadena en términos específicos, como en la figura 1(b), a otro dominio conceptual donde ciertos atributos de la cadena concreta permiten comprender la expresión. Así, inferimos que el atributo físico de la cadena, de ser una entidad con eslabones en la que cada eslabón está unido tras otro, sirve como concepto para asumir que un error -una abstracción que no es obviamente perceptible- puede ir unido a otro, y este a su vez a otro, otorgando significado a la idea de que un acto pueda provocar una cadena de errores. En este caso, la cadena de errores no es perceptible desde la corporalidad, sino que es entendida y existe gracias al mapeo o apareamiento de dominios conceptuales: UN ERROR ES UN ESLABÓN EN UNA CADENA ${ }^{1}$. En el tercer ejemplo, el dominio conceptual cadena es apareado con un dominio conceptual de las ciencias, una macromolécula orgánica -el ácido desoxirribonucleico o $\mathrm{ADN}$-. Igual que en el ejemplo anterior, hay atributos perceptibles de una cadena que se mapean o aparean con atributos imperceptibles de una entidad a ser conceptualizada: el ADN (figura 2). Así, cada unidad estructural del ADN, las bases nitrogenadas, se entienden como eslabón: UNA BASE NITROGENADA ES UN ESLABÓN EN UNA CADENA. En estos ejemplos, tanto la cadena de errores como la cadena de $A D N$ son conceptos cuya similitud es creada, o existe gracias a la comprensión del dominio conceptual físicamente perceptible cadena. A este apareamiento o creación de similitud se le llama abstracción metafórica.

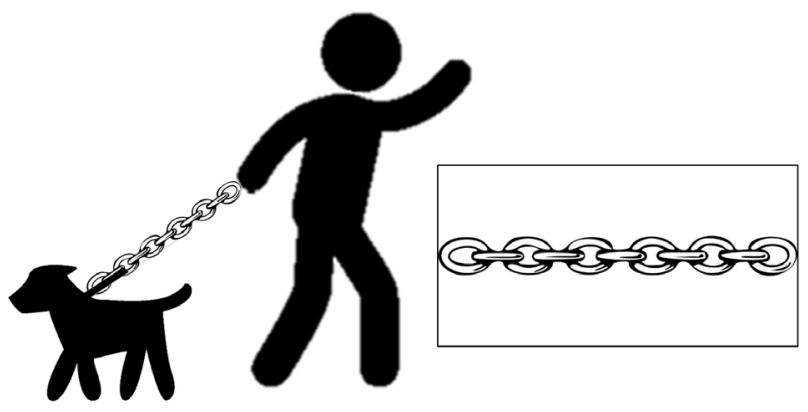

(a)

(b)

Figura 1. En la imagen (a) hay una representación de una situación cultural y fisicamente perceptible: conectar al collar de un perro una cadena para pasearlo. La entidad cadena tiene una configuración material, que está representada en (b).

Fuente: elaboración propia con imágenes liberadas.

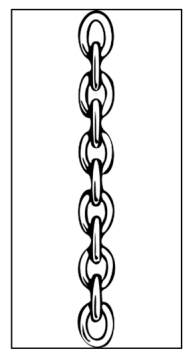

(a)

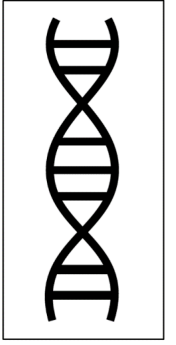

(b)

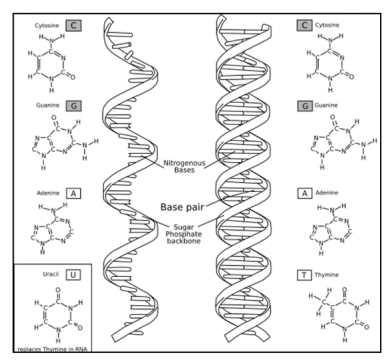

(c)

Figura 2. Representaciones de dominio conceptual cadena (a) a los dominios conceptuales de representación de la macromolécula ácido desoxirribonucleico (ADN) (b) y (c). Fuente: elaboración propia con imágenes liberadas.

1. Las metáforas conceptuales tienen una notación como la que expresa este segmento. En los siguientes párrafos se explica la notación. 
Las metáforas conceptuales se denotan usando mayúsculas, de la forma: DOMINIO META ES DOMINIO FUENTE. En los dos ejemplos anteriores, el mapeo entre dominios conceptuales queda denotado como: UN ERROR ES UN ESLABÓN EN UNA CADENA y EL ADN ES UNA CADENA (con la ya mencionada UNA BASE NITROGENADA ES UN ESLABÓN EN UNA CADENA). Para ambas metáforas, el dominio fuente es la entidad perceptible llamada cadena, y ciertos atributos de esta construyen una similitud en el concepto de error y en el concepto de $A D N$.

Lakoff y Johnson (1980) señalaron que las metáforas conceptuales son también generativas, ya que ofrecen un marco para la creación de más expresiones lingüísticas que dan cuenta de los mapeos entre los dominios conceptuales. Las siguientes expresiones, tomadas del Corpus del Español (Davies, 2017), nos permiten ejemplificar este asunto:

- Evaporación es el paso de líquido a gas.

- Todo ese superávit cognitivo que hay se va a evaporar.

- El vapor sube y en la atmósfera se transforma en nubes.

- El desempleo subió a las nubes.

El primer ejemplo ilustra un fenómeno físico que puede nombrarse y conceptualizarse (evaporación) en términos de un cambio de ubicación (o estado), en que una entidad (líquido) pasa a otra entidad (gas). La metáfora se denotaría como EVAPORAR ES MOVERSE ENTRE DOS UBICACIONES, o bien LÍQUIDO ES UNA UBICACIÓN, o bien SÓLIDO ES UNA UBICACIÓN. Con esa idea, evaporación se usa luego para conceptualizar una entidad abstracta (el superávit cognitivo), proyectando propiedades perceptuales en contexto (la idea de que algo que se evapora deja de ser visible); así, se puede proponer la metáfora SUPERÁVIT COGNITIVO ES LÍQUIDO. En el tercer ejemplo, se menciona un proceso de transferencia direccional, hacia arriba (el vapor sube), ubicando una entidad específica (nubes) que resultaría de este proceso. La percepción visual de la ubicación de las nubes sirve luego como referencia para señalar la direccionalidad de una abstracción: el desempleo. En este caso, la metáfora es MÁS ESTÁ ARRIBA, y por lo tanto orientaría el significado de la afirmación hacia un desempleo alto. Esta metáfora es generativa, dado que múltiples expresiones lingüísticas pueden crearse a partir del mapeo que se realiza entre el dominio de cantidad (MÁS) y su topología (ARRIBA). Expresiones como subió el desempleo, bajó la inflación, los precios son altos dan cuenta de la generatividad de la metáfora conceptual. La orientación vertical (arriba-abajo) muestra también que esta metáfora depende de las relaciones que establece el cuerpo con el ambiente y con las representaciones de cantidad, así como del acceso que el lenguaje en uso permite a las relaciones entre corporalidad y la creación de conceptos y dominios conceptuales. Este atributo generativo de la metáfora permite aproximarse al análisis del lenguaje en uso para poder detectar su contenido conceptual. Considerando la riqueza y despliegue conceptual necesario asociado al aprendizaje de las ciencias, el marco de metáforas conceptuales permite elaborar una perspectiva sobre los dominios conceptuales presentes en la construcción de conocimientos científicos relevantes para la educación científica y, por lo tanto, podría informar intervenciones didácticas que reconozcan este elemento analítico.

\section{Metodología}

Se realizó una revisión de la literatura desde una aproximación hermenéutica (Boell \& Cecez-Kecmanovic, 2014). La revisión hermenéutica permite un involucramiento continuo y un desarrollo gradual del corpus de literatura, con el fin de incrementar en profundidad la comprensión de una temática. Esta aproximación implica ciclos conjuntos e iterativos de búsqueda y adquisición de material bibliográfico y de análisis e interpretación de este. Los ciclos de búsqueda y adquisición incluyeron un planteamiento de búsquedas a partir de ideas iniciales, clasificaciones, selección, adquisición y lectura de materiales. 
La búsqueda se inició con el concepto de metáforas conceptuales y el trabajo pionero de Lakoff y Johnson (1980). En bases de datos especializadas (ERIC, Web of Science, Scopus), en el motor de búsquedas GoogleScholar y revistas especializadas en educación científica, se buscaron trabajos que hubieran citado la obra de Lakoff y Johnson (1980) o bien contuvieran los términos conceptual metaphor, metáfora conceptual o metáforas conceptuales en sus títulos o resúmenes hasta julio de 2018. Asimismo, en otro ciclo de búsqueda, se exploraron los mismos términos en la base de datos Scielo. En total, el primer ciclo de búsqueda arrojó 1192 coincidencias. El listado se procesó, eliminando duplicados y refinando la búsqueda para enfocarse en estudios relacionados con el aprendizaje y la enseñanza de las ciencias, o que tuvieran que ver directamente con el tema de metáforas conceptuales en las ciencias.

Siguiendo el ciclo de la aproximación hermenéutica, el material se mapeó y clasificó, realizándose un análisis crítico que permitiera entender el lugar desde donde se escribía cada trabajo; se elaboró un argumento, y con ello se formularon problemas o preguntas de investigación iniciales. Estas preguntas dieron lugar, a su vez, a nuevos ciclos de búsqueda y adquisición, permitiendo identificar y refinar las búsquedas, así como agrupar los trabajos en categorías emergentes (por ejemplo, temáticas, actores involucrados en los estudios). Las búsquedas, en términos temporales, se situaron en etapas posteriores a 1980, salvo una excepción. Se excluyeron los trabajos enfocados en analogías. Como resultado, esta revisión incluye un total de 31 trabajos, cinco de los cuales abordan la discusión de las metáforas en las ciencias y el resto, en el aprendizaje y enseñanza. La mayor parte de estos trabajos han sido publicados en revistas o fuentes de habla inglesa. Este corpus incluyó dos libros, 24 artículos en revistas indexadas, y cinco trabajos de otras fuentes.

\section{Resultados}

Al mapear y clasificar la literatura, emergieron dos categorías principales: trabajos que otorgaban a la metáfora y metáfora conceptual un rol en las ideas científicas, y trabajos que investigaban o abordaban el concepto de metáfora conceptual en la educación científica. Los resultados de la revisión están organizados con base en estas categorías: metáfora conceptual en las ciencias y metáfora conceptual en educación científica.

\section{Metáfora conceptual en las ciencias}

La noción de metáfora en las ciencias no es nueva. Los trabajos agrupados bajo esta categoría representan aquellos enfocados en discutir respecto de las ciencias y su relación con las metáforas conceptuales. Se incluye una referencia previa a 1980, dado que su contenido pudo relacionarse con el tema. Esta revisión da cuenta del tránsito de la discusión en las ciencias respecto de la incorporación del concepto de metáfora conceptual, por lo que su orden de presentación es cronológico.

A principios del siglo XX, Harris (1912) señalaba que la historia de la ciencia contaba con ejemplos en los cuales una idea era primeramente representada por una expresión metafórica para convertirse, en el tiempo, en una existencia concreta. Para Harris, el principio de la encarnación de las ideas era un proceso que ocurría en las ciencias, como aquel en el que una idea vaga era anticipada en el mundo de la mente para adquirir, en el futuro, una expresión concreta. Décadas más tarde, Hoffman (1980) analizó el rol de las metáforas en las teorías científicas, señalando que los científicos tienden a evidenciar una actitud favorable hacia ellas dada su abundante disposición en ciencias, y su aparente disponibilidad en cualquier problema al cual se enfrenten. Hoffman señaló que los científicos estarían conscientes de que las metáforas científicas y las teorías necesitan cambiar con la evidencia, ya que se usan para explorar la naturaleza, pero no se pueden confundir con la naturaleza misma. La utilidad de las metáforas en ciencias residiría en no perpetuar una distinción tan drástica entre lo literal y lo figurativo. 
Bradie (1999) señaló que las metáforas son indispensables para el pensamiento científico, y que no hay posibilidad de caracterizar la ciencia prescindiendo de ellas. Apuntó a la existencia de tres funciones de las metáforas en ciencias. La primera sería la retórica, o su uso en pedagogía y comunicaciones. La segunda sería la heurística, que corresponde a una guía para orientar nuevos descubrimientos (por ejemplo, hablar de las partículas de un gas como bolas de billar). La tercera sería la cognitiva (o teórica), contribuyendo a la validación y a la justificación de teorías.

El químico Theodore L. Brown (2003), en el libro Construyendo la verdad: metáfora en ciencia (traducción propia), vinculó metáfora conceptual con ciencias. Brown argumentó respecto de la naturaleza de las ciencias con dos ideas fundamentales: i) que los científicos comprenden la ciencia principalmente en términos de conceptos metafóricos que se basan en una comprensión corporal de cómo la naturaleza funciona, y ii) que los modelos y teorías que los científicos usan para explicar las observaciones del mundo son constructos metafóricos. Brown señaló que la comprensión de la naturaleza y de la ciencia derivan de interacciones muy básicas con el mundo físico, y que son tácitas y ampliamente compartidas por la humanidad. El entendimiento de las ciencias y la comprensión de su éxito implicarían dar cuenta de que existe un acceso a la realidad que está siempre mediado, y que la implicancia de verdad es siempre resultante del razonamiento humano (en oposición a una verdad objetiva e independiente de la mente).

Tajer (2012), médico cardiólogo, hizo una revisión de las metáforas conceptuales más relevantes en medicina, realizando un análisis que resalta la relevancia de encontrar metáforas que cambien los significados atribuidos a las metáforas existentes y su relación con el sufrimiento de los pacientes. En otro reporte, Sá, Nagem, Almeida y Marcelos (2014) estudiaron las metáforas conceptuales respecto de concepciones de evolución en dos libros: El origen de las especies de Charles Darwin y Early Man, de Francis Clark Howell. Comprobaron que, en textos de divulgación de la teoría evolutiva, la metáfora conceptual EVOLUCIÓN ES PROGRESO estaba contenida en varios ejemplos. Si bien no se encontró en el texto de Darwin, en el de Howell sí se localizaron, identificando expresiones como camino y marcha. Por último, Palma $(2008,2015)$ señaló que las metáforas en las ciencias cumplen primordialmente un rol epistémico y cognitivo. Es decir, son parte constitutiva del conocimiento científico, y también de su enseñanza.

Esta parte de la revisión permite visualizar un tránsito en la comprensión de las metáforas y su rol en el conocimiento científico, relevándolas crecientemente para entender la historia de la ciencia y la aplicación práctica a contextos, tales como el de la medicina. Los argumentos de la literatura apuntan a prestarle mayor atención a la dimensión cognitiva y epistémica de la metáfora, en contraposición a su concepción tradicional (ornamental) del lenguaje.

\section{Metáfora conceptual en la educación científica}

En esta categoría se encuentran los trabajos publicados con un foco directo en educación científica. Se comienza con una revisión realizada en 2012, para luego dar una cuenta de la evolución temporal de los estudios en los que la referencia a Lakoff y Johnson (1980) es parte informativa. Ello permite mostrar cómo ha permeado esta referencia a metáforas conceptuales en la literatura de educación científica, evidenciando también que la atención a la metáfora conceptual como fenómeno en la educación científica es una tendencia reciente.

En el trabajo titulado Comprender requiere corporalidad: un reanálisis guiado por teoría sobre el rol de las metáforas y analogías en la comprensión de la ciencia (traducción propia), Niebert, Marsch y Treagust (2012) argumentaron la importancia de las metáforas conceptuales para la enseñanza y el aprendizaje de las ciencias. El trabajo indagó analíticamente en las metáforas conceptuales a partir del lenguaje en uso en enseñanza y aprendizaje de las ciencias, con base en registros en publicaciones de educación científica. Encontraron 199 metáforas conceptuales usadas en la construcción de diversos conceptos científicos. Los autores señalaron la necesidad de ampliar la idea de lo que se considera conocimiento previo, reconociendo manifiestamente la prevalencia de experiencias corporales con el fin de facilitar la comprensión de conceptos científicos. 
Brookes y Etnika (2015) estudiaron el lenguaje en uso de científicos que enseñaban y conversaban, y estudiantes que aprendían, sobre mecánica cuántica. Concluyeron que los físicos usaban naturalmente metáforas conceptuales al hablar sobre mecánica cuántica, pero sus estudiantes tenían dificultad para comprenderlas, debido a que las interpretaban de manera literal. Por su parte, en una serie de ensayos, Fuchs (2007, 2009, 2010) desarrolló una justificación analítica para considerar experiencias corporales para la enseñanza de la física y química, argumentando que la lingüística cognitiva ofrece herramientas para analizar los conceptos científicos que traen los estudiantes. Fuchs enfatizaba el estudio de fenómenos lingüísticos como las metáforas conceptuales, la dinámica de fuerzas y los esquemas de imagen ${ }^{2}$, dado que estarían interrelacionados a propósito de la relación del cuerpo con el ambiente, que representaría una fuente de conceptualizaciones clave para la física y dinámica de fluidos. Nehm, Rector y Ha (2010) analizaron, mediante un sistema computarizado de evaluación, la frecuencia con la que estudiantes de biología en la universidad usaban palabras asociadas con términos de dinámica de fuerzas con el fin de explorar la precisión o imprecisión de sus explicaciones respecto de la biología evolutiva. Sus conclusiones llaman la atención de los educadores científicos sobre el uso del habla de fuerza con el fin de explicar modelos de evolución biológica, dado que llevarían a explicaciones incorrectas. La dinámica de fuerzas es considerada como un discurso primario que tienen las y los estudiantes que aprenden ciencia, que serviría para ubicar sus desempeños en una línea de progresión hacia modelos más sofisticados de razonamiento científico (Gunckel, Covitt, Salinas, \& Anderson, 2012; Gunckel, Mohan, Covitt, \& Anderson, 2012; Mohan, Chen, \& Anderson, 2009).

Dreyfus y sus colegas (2014) estudiaron metáforas ontológicas para el concepto de energía en el aprendizaje de la física, identificando dos metáforas para la energía: una de ubicación y otra de sustancia. Señalaron la naturaleza problemática de la metáfora de sustancia cuando se habla de energía negativa en reacciones químicas, y argumentaron que una aproximación interdisciplinaria a la comprensión de energía, en la que se mezclen ambas metáforas, puede ser fructífera para razonar acerca de fenómenos como la energía negativa en los enlaces químicos. Por su parte, Lancor (2014) realizó un estudio del concepto de energía en química, física y biología mediante metáforas conceptuales. Se enfocó en lo que llamó discurso pedagógico, o lo escrito en libros de texto y literatura de educación científica. Logró identificar seis metáforas de sustancia para referirse al concepto de energía: energía como sustancia que puede ser cuantificada, que puede fluir, que puede cambiar de forma, que puede perderse, y que puede ser un ingrediente, un producto, o puede guardarse de alguna forma. Para cada metáfora hay aspectos que son destacados, mientras otros son ocultados. Por ejemplo, la metáfora de energía como sustancia que puede fluir destaca la idea de una fuente de energía, pero oculta la de transformación de energía. Por otro lado, la metáfora de la energía como sustancia que puede ser cuantificada destaca la idea de la conservación de la energía, mientras oculta la de su fuente de transformación. Lancor señaló que no hay una metáfora conceptual única que permita explicar el concepto complejo y abstracto de energía en su completitud.

Niebert y Gropengießer (2014) realizaron un análisis metafórico al contenido de 35 entrevistas a estudiantes de secundaria respecto de sus comprensiones sobre el efecto invernadero, y analizaron reportes de investigación y libros de texto sobre el mismo tema. Señalaron que las concepciones de científicos y estudiantes se basan en los mismos esquemas, siendo tres concepciones las fundamentales: calentamiento por más aporte, calentamiento por menos aporte, y calentamiento por un nuevo equilibrio. Esta interrelación entre las concepciones de estudiantes y científicos les permite describir algunas demandas para el aprendizaje de los estudiantes respecto del tema, indicando algunos principios para diseños de experiencia de aprendizaje sobre el efecto invernadero, donde los estudiantes puedan explorar las interacciones de las radiaciones electromagnéticas y dióxido de carbono, y reflexionar sobre las experiencias que usan como dominios fuente para la comprensión metafórica del efecto invernadero.

2.Tanto la dinámica de fuerzas como los esquemas de imagen son fenómenos lingüísticos también muy relevantes en el campo de la lingüística cognitiva. Son importantes en la formulación de dominios que constituyen metáforas conceptuales. Si bien están relacionados de forma importante con la metáfora conceptual, son atendidos brevemente en este artículo. Para referencias sobre la dinámica de fuerzas ver Alarcón (2010) y, sobre esquemas de imagen, ver Ibarretxe-Antuñano y Valenzuela (2012). 
En otro trabajo, Salinas (2014) exploró, mediante análisis de metáforas conceptuales, las experiencias corporales y perceptuales en las que se basan estudiantes de escuela secundaria para conceptualizar la idea de sistema. El autor estableció que se puede inferir que las percepciones respecto de los límites de las entidades físicas y la dinámica de percepción sobre los cambios de posición (locomoción) son puntos de partida, o dominios fuente, para el desarrollo del pensamiento sistémico como dominio meta.

La edición especial de la revista International Journal of Science Education en 2015, titulada Metáfora conceptual y cognición corpórea en el aprendizaje de las ciencias (traducción propia) presentó siete trabajos -algunos de cuyos autores ya emergían publicando respecto del tema- y tres comentarios, confirmando la creciente atención al estudio de las metáforas conceptuales en la investigación en educación científica en contextos angloparlantes. Amin, Jeppsson y Haglund (2015) presentaron la edición especial, considerando el énfasis en metáfora conceptual como una categoría útil en el estudio de fenómenos del aprendizaje de las ciencias. Brookes y Etnika (2015) reportaron que entrevistaron a estudiantes de física de nivel universitario para explorar su razonamiento respecto del calor en procesos termodinámicos. El estudio reveló algunas relaciones entre la forma en la que los estudiantes comprendían el calor y su aproximación a resolver problemas del calor, aportando a describir los efectos de considerar la metáfora del calor como sustancia en la comprensión de fenómenos termodinámicos.

Jeppsson, Haglund y Amin (2015) reportaron que el uso de metáforas conceptuales en la interpretación del conocimiento proposicional, tanto lingüístico como matemático, sería un aprendizaje que está en la base del desarrollo de la experticia científica aplicada a la resolución de problemas. Por su parte, Dreyfus, Gupta y Redish (2015) continuaron su elaboración respecto de la productividad conceptual de usar distintas metáforas para el concepto de energía. En su análisis, el uso común de metáforas ontológicas como LA ENERGÍA ES UNA SUSTANCIA y LA ENERGÍA ES UNA UBICACIÓN VERTICAL pueden ser mezcladas para generar, tanto en novatos como en expertos, un modelo mental coherente que permite razonar en torno al concepto de energía. Close y Scherr (2015) reportaron el efecto del uso de estas mezclas, ilustrando la creación de un espacio de aprendizaje para la comprensión de los fenómenos de transferencias y transformaciones de energía. El espacio de aprendizaje implica interacciones de cada aprendiz, como movimiento corporal, gestual, y habla metafórica, con la metáfora de LA ENERGÍA ES UNA SUSTANCIA. Los autores mostraron que los aspectos mezclados del ambiente de aprendizaje promueven un involucramiento intelectual activo con la comprensión del concepto de energía. Así, estiman que hay metáforas conceptuales específicas que pueden mezclarse con una actividad humana concreta y que pueden ser usadas para beneficiar la enseñanza de las ciencias. Lancor (2015) expandió su estudio sobre metáforas de energía hacia el discurso de estudiantes de nivel universitario, realizando un análisis cualitativo para estudiar las metáforas que emergieron cuando solicitaron a 49 estudiantes de un curso general de ciencias explicar el concepto de energía en cinco contextos: radiación, transporte, generación de electricidad, terremotos, y la teoría del big-bang. La mayor parte de los estudiantes usaron metáforas conceptuales múltiples y coherentes para explicar el rol de la energía en distintos contextos, no solo los tradicionalmente científicos.

Niebert y Gropengiesser (2015) expandieron la noción del fundamento experiencial del pensamiento científico -usando metáforas conceptuales- para comprender las bases corporales de ciertas concepciones científicas. Estudiaron cuáles son las concepciones corporales que estudiantes y científicos aprovechan para comprender fenómenos representados en el microcosmos y el macrocosmos, y cómo estas concepciones permitirían el diseño de representaciones externas de fenómenos microcósmicos y macrocósmicos. Los autores argumentan que el acceso de nuestra percepción, como humanos, está adaptado a una dimensión de mediana escala, y nuestras concepciones corporales se originan en esa escala mesocósmica. Identificaron en este estudio variadas metáforas conceptuales para comprender fenómenos microcósmicos, como el crecimiento microbial y la conducción de señales neuronales, y fenómenos macrocósmicos, como el efecto invernadero y el ciclo del carbono. 
Fuchs (2015) discutió sobre metáforas conceptuales y su vínculo con el campo de estudios de narrativas en el aprendizaje de las ciencias. En su trabajo, propuso la idea del marco narrativo para escenarios naturales y técnicos, con el fin de representar el apresto de la inteligencia narrativa en la percepción de fenómenos, y relatos que contengan elementos conceptuales usados para modelar fenómenos científicos. Fuchs se apoyó en el estudio analítico de historias simples de fenómenos naturales y de productos asociados a la termodinámica. En el estudio, reconoce lo que llama estructuras figurativas, mostrando que la idea perceptual de FUERZA DE LA NATURALEZA (énfasis en el original) se basa en una estructura narrativa, donde se asume que agentes naturales actúan y sufren en mundos relatados. Fuchs argumentó que el pensamiento científico y sus modelos formalizados están fuertemente ligados a estos mundos relatados, que contienen y deben estar formados por agentes, tensiones, eventos y procesos, causalidad y relaciones de poder, y una conexión a una comprensión emocional. Para el autor, si el mundo narrado ha de contribuir al pensamiento científico, debe tener además cierto grado de estructura conceptual, dada por las proyecciones metafóricas, que permita formalizar cierto pensamiento científico.

Celik (2016) estudió la percepción de 226 estudiantes de cuatro niveles educativos de enseñanza secundaria en Turquía sobre fenómenos de calor, temperatura y energía, logrando reconocer 176 metáforas generadas por las y los estudiantes participantes, mediante evaluaciones o instrumentos de provocación de metáforas. Las metáforas generadas por los estudiantes fueron clasificadas como metáforas formales/científicas, metáforas abstractas, metáforas ambientales/cotidianas, y metáforas de concepciones erróneas. El autor ordenó las metáforas y sus clasificaciones en términos de su prevalencia creciente en cada uno de los niveles educativos, sugiriendo la existencia de diferencias o etapas de las metáforas. Celik discute la posibilidad de avanzar en construir modelos de análisis de estas a partir de lo que señalan los estudiantes para crear modelos de enseñanza de conceptos científicos clave.

Kersting y Steier (2018) estudiaron, mediante un análisis temático y metafórico, las explicaciones escritas en línea en pequeños grupos de estudiantes universitarios de sus clases de física en Noruega. El tema era la naturaleza abstracta del tiempo espacial. Los investigadores encontraron que los estudiantes generaban metáforas conceptuales que estaban reportadas en la literatura, así como también otras nuevas que llevan a concepciones distintas sobre la gravedad respecto de aquellas de los expertos en el campo. Los autores identificaron un conflicto de comprensión entre lo que los estudiantes entienden como gravedad en términos corporales y la descripción abstracta sobre relatividad general. A partir de estos hallazgos, los investigadores ofrecieron algunas orientaciones para la enseñanza de la relatividad general, también asumiendo las implicancias epistemológicas del uso de metáforas conceptuales específicas en las aulas escolares.

La revisión de esta literatura otorga una panorámica de la influencia que ha tenido el estudio de las metáforas conceptuales en la comprensión de fenómenos científicos, así como también en el aprendizaje y enseñanza de las ciencias. Resumiendo en general los resultados de la revisión, se puede afirmar que:

- Existe un aparente y creciente interés por atender el fenómeno de las metáforas conceptuales en las ciencias y en los procesos de la educación científica.

- Las metáforas conceptuales pueden identificarse en variadas áreas de la educación científica y sobre diversos conceptos, lo que implica un repositorio aún no sistemático de metáforas en uso en educación científica.

- Las y los investigadores tienden a reconocer las metáforas en distintos niveles educativos, a distintos niveles de experticia científica, y en distintos materiales y contextos de uso de lenguaje científico, pudiendo o no coincidir para un mismo concepto.

- Hay limitados ejemplos de uso de metáforas conceptuales para el diseńo de experiencias de enseñanza de las ciencias, ubicados casi en su totalidad en contextos de habla inglesa. 
La revisión muestra que, en algunos contextos internacionales, existe investigación que tiende a converger respecto de la importancia de la corporalidad y el estudio de las metáforas conceptuales en la comprensión de conceptos científicos. También muestra que es un campo incipiente, con trabajos recientes. A continuación, se discuten los resultados de esta revisión como aporte a una agenda de investigación básica y aplicada a la educación científica en contextos hispanohablantes.

\section{Discusión y conclusiones}

Tanto el interés emergente por el estudio de las metáforas conceptuales, su identificación en contextos de comunicación y educación científica, y el reconocimiento de su uso diferenciado en distintos contextos y a distintos niveles permite ordenar sus potencialidades. Asimismo, el desarrollo del campo de la lingüística cognitiva en habla hispana también presenta una oportunidad para avanzar en una perspectiva respecto del rol de las metáforas en la educación científica a nivel latinoamericano. Esta discusión refiere a la propuesta de investigación básica -la potencialidad epistémica- y de investigación aplicada -la potencialidad didáctica- del estudio de las metáforas conceptuales para la educación científica.

De cierta forma, la revisión señala que hay un campo de estudios en el que la investigación sistemática sobre metáforas conceptuales, entendidas como fenómeno del lenguaje que permite acceso a patrones de corporalidad y comprensión conceptual, puede ser llevada a cabo en contextos de educación científica.

\section{Agenda de investigación básica}

El interés de la comunidad internacional respecto del estudio de las metáforas conceptuales en la educación científica puede explicarse con el tránsito que han tenido algunas perspectivas sobre el lenguaje en las últimas décadas, y el modo en que se han vinculado con los estudios sobre aprendizaje, específicamente con el desarrollo del campo de la lingüística cognitiva (Evans \& Green, 2006; Geeraerts \& Cuyckens, 2007; Lee, 2001; Ungerer \& Schmid, 1996). Si bien ha llegado a la comunidad de educación científica angloparlante, a nivel hispanoparlante existe un desarrollo de la lingüística cognitiva más reciente (Ibarretxe-Antuñano y Valenzuela, 2012), lo que explica en parte que el interés se haya desarrollado con más tardanza. Esto representa una oportunidad para pensar en los estudios del lenguaje en educación científica desde las metáforas conceptuales, proyectando el estudio del lenguaje en las ciencias más allá de las aproximaciones que se preocupan de buscar las distinciones que tiene el lenguaje científico frente a otros discursos (Gee, 2005). Al propender a una visión del lenguaje como una facultad inseparable de la cognición, y por tanto una ventana hacia el pensamiento (Pinker, 2007), se pueden estimular investigaciones que identifiquen en el lenguaje científico patrones de pensamiento vinculados con otras experiencias, eventualmente significativas en la construcción de los conceptos científicos. Las metáforas conceptuales permiten al pensamiento científico mirar a través de esas ventanas, buscando discernir cuáles serían aquellos dominios fuente, o experiencias base de los conceptos científicos.

La revisión da cuenta del esfuerzo investigativo asociado con la búsqueda de los dominios fuente en torno a distintos conceptos científicos. Para continuar con la identificación de estos dominios fuente y su vínculo con conceptos científicos, se requieren acuerdos sobre el uso de métodos, formas de análisis, y fuentes de datos que permitirían sistematizar este trabajo y desarrollarlo. Esto implica un diálogo metodológico en el campo de los estudios de educación científica, para precisar y discernir cuáles serían los dominios conceptuales relevantes y cómo podrían denotarse y ejemplificarse para futuras investigaciones. Además, se requiere de una profundización en el diálogo interdisciplinar sobre el fenómeno de las metáforas desde la perspectiva lingüística, tal como lo ha planteado Steen (2011) en la propuesta revisitada de la teoría contemporánea de metáfora que enfatiza su dimensión comunicativa. 
La distinción de las metáforas conceptuales con base en los niveles y contextos de uso puede también otorgar una productiva discusión al campo de estudios sobre el aprendizaje de las ciencias. Un alcance de esta línea de investigación puede significar nuevas discusiones epistemológicas en torno a las formas en las que la didáctica de las ciencias se permite entender conceptos incorporados hoy a la búsqueda de experiencias de aprendizaje, como el cambio conceptual. Niebert y sus colegas (Niebert \& Gropengiesser, 2015; Niebert \& Gropengießer, 2014; Niebert et al., 2012) han invitado a considerar el experiencialismo como marco teórico para reexaminar nuestras concepciones sobre cómo el aprendizaje ocurre en las ciencias. Asimismo, proponen pasar desde la ejemplificación de las ideas previas de los estudiantes hacia explicaciones que señalen el porqué de esas ideas previas en primer lugar. Por su parte, Amin $(2009,2015)$ ha argumentado que las metáforas conceptuales pueden ser ubicadas como fuentes adicionales dentro del estudio del cambio conceptual. Por su parte, Palma $(2008,2015)$ apuntó a que la metáfora tiene un rol cognoscitivo y epistémico fundamental, tanto para la producción de conocimiento científico como en la apropiación que de este hacen quienes aprenden ciencia. Se propuso "analizar la naturaleza y función de las metáforas para comprender el tipo de compromisos conceptuales, intelectuales y epistemológicos que se asumen cuando se las enuncia y aprovechar sus potencialidades" (Palma, 2008, p. 17). Esta visión podría promover otras perspectivas sobre el progreso del aprendizaje en las ciencias, y con ello dinamizar la discusión curricular.

Los resultados de esta revisión permiten señalar la potencialidad de desarrollar una línea de estudios en educación científica que apunte a vincular el examen del lenguaje en uso en contextos científicos y de aprendizaje y enseñanza desde la comprensión de las metáforas conceptuales.

\section{Potencialidades didácticas}

El creciente interés por el estudio de las metáforas conceptuales en educación científica está cruzado por algunas intervenciones en aspectos pedagógicos o didácticos. En la mayor parte de los artículos analizados, hay intención de describir las metáforas conceptuales asociadas a conceptos científicos, y entender su uso en contextos escolares o universitarios. Por ejemplo, Niebert y sus colegas sugirieron que un "análisis de los antecedentes experienciales de las concepciones cotidianas y científicas puede resultar en una base fructífera para el desarrollo de ambientes de aprendizaje” (p. 23, traducción propia). No obstante, el interés se expresa más en contextos angloparlantes, mientras que en contextos hispanoparlantes se ha reconocido el valor epistémico de las metáforas en las ciencias, y por tanto su valor para la enseñanza de las ciencias (Palma 2008, 2015). Esto supone un desafío para el campo, por elaborar y entender la potencialidad pedagógica y didáctica del estudio de las metáforas conceptuales en la educación científica. Desde esa perspectiva, las metáforas conceptuales se han usado para proponer aplicaciones didácticas en el aprendizaje de una segunda lengua (Hijazo-Gascón, 2011), y en vínculo con perspectivas comunicativas (Steen, 2011) y consideraciones de la ciencia como una segunda lengua (Lemke, 1990; Darian, 2003; Roth, 2005; Reeves, 2005; Bruna \& Gómez, 2009) se podrían proponer elementos de experiencia para desarrollar propuestas didácticas asociadas a las metáforas conceptuales.

La identificación de las metáforas conceptuales en conceptos científicos podría aportar enormemente al diseño de experiencias de aprendizaje de las ciencias. En este trabajo, se han presentado, por ejemplo, diversas metáforas para referirse al concepto de energía. Estos ya constituyen un recurso o repositorio de dominios conceptuales fuente, que construyen y le dan existencia al concepto científico, o dominio conceptual meta, de energía. Al identificar más de esos dominios conceptuales fuente, se contará con herramientas para contribuir a diseñar experiencias que sean fácilmente vinculables a la comprensión del dominio meta. Esta exploración está abierta.

Varios de los trabajos acá presentados han examinado las metáforas que se encuentran presentes en distintos contextos y niveles, y que son usadas en el lenguaje de diferentes actores con diversos roles en el contexto de la educación científica. Este hallazgo combina la posibilidad de entender un aspecto desarrollista o progresivo de la metáfora conceptual, o bien uno en el que el contexto genere una influencia para su comprensión. Al permitirse 
entender las metáforas en distintos contextos, se puede establecer la utilidad de los materiales curriculares para diferentes actores y roles. Preguntas como, ¿qué metáforas están a la base de los conceptos científicos a enseñar?, o bien ¿cuál podría ser la metáfora más relevante a usar en el contexto o nivel en el que se enseña? podrían motivar innovaciones que, hasta lo ahora revisado, han sido limitadamente informadas en la literatura.

En esta revisión se muestran algunos ejemplos de investigaciones que avanzan aplicando lo que aprenden sobre metáforas conceptuales al diseño de estas experiencias de aprendizaje (Lancor, 2014; Niebert \& Gropengießer, 2014; Close \& Scherr, 2015). Así, los elementos interesantes de avance en el campo de la didáctica de las ciencias con esta orientación podrían ser el estudio experimental o cuasi experimental de intervenciones didácticas basadas en estas investigaciones sobre metáforas conceptuales. Es decir, el comprender metafóricamente, desde el experiencialismo, algunos conceptos científicos clave que organicen en parte el currículo, podría promover innovaciones didácticas cuyo impacto puede entenderse también desde la forma en que se vinculan los dominios fuente con los dominios meta. El alcance posible de estos estudios podría entregar al campo nuevos marcos de organización, o bien, resituar algunos elementos del constructivismo en la enseńanza de las ciencias, como la noción de ideas previas al aprendizaje de conceptos científicos. El estudio sistemático de las metáforas conceptuales podría potenciar el interés de la comunidad hispanoparlante en experimentar con ellas para diseñar experiencias didácticas. Algo de esta innovación se ha presentado en Salinas, González y Fernández (2017).

La revisión acá presentada llama a reexaminar el lenguaje de las ciencias desde la perspectiva del experiencialismo, que entiende al conocimiento humano abstracto como un resultado de la experiencia con dominios conceptuales fuente, en particular la experiencia corporal con el ambiente. La metáfora conceptual como fenómeno lingüístico captura el vínculo entre dominios conceptuales de la experiencia. Hay quienes argumentan que la metáfora no solo es sobre el pensamiento, sino también sobre la comunicación, lo que permite abrir la discusión respecto de cómo aproximarse al fenómeno metafórico desde una perspectiva social (Steen, 2011). Este trabajo se ha limitado a revisar la investigación asociada con el concepto de metáforas conceptuales en la educación científica, que ha resonado fundamentalmente en la investigación reportada en inglés, y que argumenta que la comprensión de conceptos científicos requiere de corporalidad. Considerando esa información, se propone que el campo de la educación científica preste atención a este concepto, estimulando su potencial para comprender condiciones para informar y estudiar innovaciones en el área.

El artículo original fue recibido el 19 de diciembre de 2019

El artículo revisado fue recibido el 20 de septiembre de 2020

El artículo fue aceptado el 29 de septiembre de 2020

\section{Referencias}

Alarcón, P. (2010). Dinámica de Fuerzas y Metáfora Conceptual en "Pasarse de Largo." Un Estudio en Lingüística Cognitiva. Onomázein, 22(2), 107-124. Recuperado de http://onomazein.letras.uc.cl/Articulos/22/5_Alarcon.pdf

Amin, T. G. (2009). Conceptual metaphor meets conceptual change. Human Development, 52(3), 165-197. https://doi.org/10.1159/000213891

Amin, T. G. (2015). Conceptual Metaphor and the Study of Conceptual Change : Research synthesis and future directions Conceptual Metaphor and the Study of Conceptual Change : Research synthesis and future directions. International Journal of Science Education, 37(5-6), 966-991. https://doi.org/10.1080/09500693.2015.1025313

Amin, T. G., Jeppsson, F., y Haglund, J. (2015). Editorial Material: Conceptual Metaphor and Embodied Cognition in Science Learning: Introduction to special issue. International Journal of Science Education, 37(5-6), 745-758. https://doi.org/http://dx.doi.org/10.1080/09500693.2015.1025245 
Boell, S. K. \& Cecez-Kecmanovic, D. (2014). A hermeneutic approach for conducting literature reviews and literature searches. Communications of the Association for information Systems, 34, 257-286. https://doi.org/10.17705/1CAIS.03412

Bradie, M. (1999). Science and metaphor. Biology and Philosophy, 14, 159-166. https://doi.org/10.1023/A:1006601214943

Brookes, D. T. \& Etkina, E. (2015). The Importance of Language in Students' Reasoning About Heat in Thermodynamic Processes. International Journal of Science Education, 37(5-6), 759-779. https://doi.org/10.1080/09500693.2015.1025246

Brown, T. L. (2003). Making truth: Metaphor in science. Urbana, IL: University of Illinois Press.

Bruna, K. R. \& Gomez, K. (2009). The work of language in multicultural classrooms: Talking science, writing science. Nueva York, NY: Routledge.

Celik, H. (2016). An Examination of Cross Sectional Change in Student's Metaphorical Perceptions towards Heat, Temperature and Energy Concepts. International Journal of Education in Mathematics, Science and Technology, 4(3), 229-245. Recuperado de https://www.ijemst.net/index.php/ijemst/article/view/90

Close, H. G. \& Scherr, R. E. (2015). Enacting Conceptual Metaphor through Blending: Learning activities embodying the substance metaphor for energy. International Journal of Science Education, 37(5-6), 839-866. https://doi.org/10.1080/09500693.2015.1025307

Darian, S. (2003). Understanding the language of science. Austin, TX: University of Texas Press.

Davies, M. (2017). Corpus del Español: Two billion words, 21 countries. Recuperado desde http://www.corpusdelespanol.org/web-dial/

Dreyfus, B. W., Geller, B. D., Gouvea, J., Sawtelle, V., Turpen, C., \& Redish, E. F. (2014). Ontological metaphors for negative energy in an interdisciplinary context. Physical Review Special Topics - Physics Education Research, 10, 02108. https://doi.org/10.1103/PhysRevSTPER.10.020108

Dreyfus, B. W., Gupta, A., \& Redish, E. F. (2015). Applying Conceptual Blending to Model Coordinated Use of Multiple Ontological Metaphors. International Journal of Science Education, 37(5-6), 812-838. https://doi.org/10.1080/09500693.2015.1025306

Evans, V. \& Green, M. (2006). Cognitive linguistics: An introduction. Edinburgh, Reino Unido: Edinburgh University Press.

Fuchs, H. U. (2007). From image schemas to dynamical models in fuids, electricity, heat, and motion. An essay on physics education research. Recuperado de http://www.hansfuchs.org/COURSES/JO/Files_V/PER_Essay.pdf

Fuchs, H. U. (2009). Figurative structures of thought in science. En An Evolutionary Cognitive Perspective on Science Learning Talk presented to the General Assembly of the Conférence des directeurs de gymnase de Suisse Remande et $d u$ Tessin. Mendrisio. Recuperado de http://www.hansfuchs.org/MATERIALS/Mendriso_Talk.pdf

Fuchs, H. U. (2010). Force dynamic gestalt, metaphor, and scientific thought. En Invited Talk at the Conference "Innovazione nella didattica delle scienze nella scuola primaria: al crocevia fra discipline scientifiche e umanistiche "Università degli studi di Modena e Reggio Emilia (pp. 12-13).

Fuchs, H. U. (2015). From Stories to Scientific Models and Back: Narrative framing in modern macroscopic physics. International Journal of Science Education, 37(5-6), 934-957. https://doi.org/10.1080/09500693.2015.1025311

Geary, J. (2011). I is an other: The secret life of metaphor and how it shapes the way we see the world. Nueva York, NY: Harper Collins Publishers.

Gee, J. P. (2005). Language in the science classroom: Academic social languages as the heart of school-based literacy. En R. K. Yerrik \& W. Roth (Eds.), Establishing scientific classroom discourse communities: multiple voices of teaching and learning research (pp. 19-37). Mahwah, NJ: Lawerence Erlbaum Associates.

Geeraerts, D. \& Cuyckens, H. (2007). The Oxford handbook of cognitive linguistics. Oxford, MS: Oxford University Press.

Gunckel, K. L., Covitt, B. A., Salinas, I., \& Anderson, C. W. (2012). A learning progression for water in socio-ecological systems. Journal of Research in Science Teaching, 49(7), 843-868. https://doi.org/10.1002/tea.21024

Gunckel, K. L., Mohan, L., Covitt, B. A., \& Anderson, C. W. (2012). Addressing challenges in developing learning progressions for environmental science literacy. En A. Alonzo y A. W. Gotwals (Eds.), Learning Progresions in Science (pp. 39-75). Rotterdam, Holanda: Sense Publishers.

Harris, D. F. (1912). The Metaphor in Science. Science, 36(922), 263-269. Recuperado de https://www.jstor.org/stable/1637398 
Hijazo-Gascón, A. (2011). Las metáforas conceptuales como estrategias comunicativas y de aprendizaje: Una aplicación didáctica de la lingüística cognitiva. Hispania 94(1), 142-154. Recuperado de https://www.jstor.org/stable/23032090?seq=1

Hoffman, R. R. (1980). Metaphor in Science. En R. P. Honeck y R. R. Hoffman (Eds.), The psycholinguistics of figurative language (pp. 393-423). Hillsdale, NJ: Erlbaum.

Ibarretxe-Antuñano, I. y Valenzuela, J. (2012). Lingüística Cognitiva: Origen, Principios y Tendencias. En I. Ibarretxe-Antuñano \& J. Valenzuela (Eds.), Lingüistica Cognitiva (pp. 13-38). Barcelona, Espańa: Anthropos.

Jeppsson, F., Haglund, J., \& Amin, T. G. (2015). Varying Use of Conceptual Metaphors across Levels of Expertise in Thermodynamics. International Journal of Science Education, 37(5-6), 780-805. https://doi.org/10.1080/09500693.2015.1025247

Kersting, M. \& Steier, R. (2018). Understanding Curved Spacetime: The Role of the Rubber Sheet Analogy in Learning General Relativity. Science \& Education, 27, 593-623. https://doi.org/10.1007/s11191-018-9997-4

Lakoff, G. (1987). Women, Fire, and Dangerous Things: What Categories Reveal about the Mind. Chicago, IL: The University of Chicago Press.

Lakoff, G. (1993). The contemporary theory of metaphor. En A. Ortony (Ed.), Metaphor and thought (pp. 202-251). Nueva York, NY: Cambridge University Press.

Lakoff, G. (2008). The Neural Theory of Metaphor. En J. Raymond W. Gibbs (Ed.), The Cambridge Handbook of Metaphor and Thought. Nueva York, NY: Cambridge university Press.

Lakoff, G. \& Johnson, M. (1980). Metaphors we live by. Chicago, IL: The University of Chicago Press.

Lakoff, G. \& Johnson, M. (1999). Philosophy in the flesh: the embodied mind and its challenge to western thought. Nueva York, NY: Basic Books.

Lancor, R. (2014). Using Metaphor Theory to Examine Conceptions of Energy in Biology, Chemistry, and Physics. Science \& Education, 23, 1245-1267. https://doi.org/10.1007/s11191-012-9535-8

Lancor, R. (2015). An Analysis of Metaphors Used by Students to Describe Energy in an Interdisciplinary General Science Course. International Journal of Science Education, 37(5-6), 876-902. https://doi.org/10.1080/09500693.2015.1025309

Lee, D. (2001). An introduction to cognitive linguistics. Melbourne, Australia: Oxford University Press.

Lemke, J. L. (1990). Talking science: language, learning, and values. Westport, CT: Ablex Publishing Corporation.

Mohan, L., Chen, J., \& Anderson, C. W. (2009). Developing a multi-year learning progression for carbon cycling in socioecological systems. Journal of Research in Science Teaching, 46(6), 675-698. https://doi.org/10.1002/tea.20314

Nehm, R. H., Rector, M. A., \& Ha, M. (2010). "Force-Talk" in evolutionary explanation: metaphors and misconceptions. Evolution: Education and Outreach, 3, 605-613. https://doi.org/10.1007/s12052-010-0282-5

Niebert, K. \& Gropengießer, H. (2014). Understanding the Greenhouse Effect by Embodiment - Analysing and Using Students' and Scientists' Conceptual Resources. International Journal of Science Education, 36(2), 277-303. https://doi.org/10.1080/09500693.2013.763298

Niebert, K. \& Gropengiesser, H. (2015). Understanding Starts in the Mesocosm: Conceptual metaphor as a framework for external representations in science teaching. International Journal of Science Education, 37(5-6), 903-933. https://doi.org/10.1080/09500693.2015.1025310

Niebert, K., Marsch, S., \& Treagust, D. F. (2012). Understanding needs embodiment: A theory-guided reanalysis of the role of metaphors and analogies in understanding science. Science Education, 96(5), 849-877. https://doi.org/10.1002/sce.21026

Palma, H. A. (2008). Metáforas y modelos científicos: El lenguaje en la enseñanza de las ciencias. Buenos Aires, Argentina: Libros del Zorzal.

Palma, H. A. (2015). Ciencia y metáforas: Los viejos ruidos ya no sirven para hablar. Cuadernos de NeuropsicologíaPanamerican Journal of Neuropsychology, 9(1), 134-146. Recuperado de https:/www.cnps.cl/index.php/cnps/ article/view/182

Pinker, S. (2007). The stuff of thought: language as a window into human nature. Nueva York, NY: Penguin Group.

Pinker, S. (2010). The cognitive niche: coevolution of intelligence, sociality, and language. Proceedings of the National Academy of Sciences of the United States of America, 107(Supplement 2), 8993-8999. https://doi.org/10.1073/ pnas.0914630107 
Reeves, C. (2005). The language of science. (Intertext). Nueva York, NY: Routledge.

Roth, W. M. (2005). Talking Science: Language and Learning in Science Classrooms. Lanham, MD: Rowman \& Littlefield Publishers.

Sá, N. L., Nagem, R. L., Almeida, M. G., \& Marcelos, M. F. (2014). Metáfora Marcha do progresso e as concepçôes de evolução nas obras A origem das espécies (Charles Darwin) e Early man (F. Clark Howell). Latin American Journal of Science Education, 1, 23001. Recuperado de https://lajse.org/nov14/23001_Natalia.pdf

Salinas, I. (2014). Embodied experiences for science learning: a cognitive linguistics exploration of middle school students' language in learning about water (Tesis doctoral). Recuperado de https://repository.arizona.edu/handle/10150/332761

Salinas, I., González, N., y Fernández, L. (2017). Indagación Narrativa de Aula: Casos de innovación en educación científica. Concepción, Chile: Escaparate.

Steen, G. J. (2011). The contemporary theory of metaphor — now new and improved! Review of Cognitive Linguistics, 9(1), 26-64. https://doi.org/10.1075/rcl.9.1.03ste

Tajer, C. D. (2012). Thinking Medicine Metaphorically. Revista Argentina de Cardiologia, 80(6), 485-493.

Ungerer, F. \& Schmid, H.-J. (1996). An introduction to cognitive linguistics. Nueva York, NY: Addison Wesley Longman. 